\title{
Diagnostic Challenges in Right lliac Fossa Mass Caused by a Fish Bone
}

\author{
Ahmad Ibrahim Yahaya, ${ }^{1}$ Ismail Burud, ${ }^{2}$ Jasiah Zakariaa ${ }^{1}$ \\ ${ }^{1}$ Department of General Surgery, Hospital Tuanku Ja'afar, Jalan Rasah, 70300 Seremban, Negeri \\ Sembilan, Malaysia \\ ${ }^{2}$ Department of Surgery, International Medical University, Clinical Campus, 70300 Seremban, Negeri \\ Sembilan, Malaysia
}

Correspondence to: Dr. Ahmad Ibrahim Yahaya; email: aiyqosim@yahoo.com

Received: 07 December 2019; Revised: 01 June 2020; Accepted: 05 June 2020; Available online: 22 June 2020

\section{Summary}

Right iliac fossa (RIF) mass is a common condition seen by surgeons. Despite advances in diagnostic modalities, it remains a diagnostic and therapeutic challenge, hence many authors describe RIF mass as temple of surprises. We report a challenging case of a 35-year-old man who presented with a tender RIF mass. Abdominal ultrasonography (USG) and computed tomography (CT) scan abdomen were done and he was treated nonsurgically. His symptoms recurred after one month and a CT scan abdomen was repeated which revealed a suspicious foreign body within the appendicular mass. Laparotomy was performed which showed a macerated appendix with a 4-cm long fish bone within. The role of diagnostic tools in managing RIF mass and the treatment modality of appendicular mass are discussed. In managing RIF mass, a surgeon must be aware of the various differential diagnoses, but common diagnosis should always be entertained. Multi-modal diagnostic tools must be considered, including serial imaging in different planes.

Keywords: Appendicitis, Right iliac fossa mass, Fish bone

Ann Afr Surg. 2021; 18(1):59-62

DOI: http://dx.doi.org/10.4314/aas.v18i1.11

\section{Conflicts of Interest: None}

\section{Funding: None}

(C) 2021 Author. This work is licensed under the Creative Commons Attribution 4.0 International License.

\section{Introduction}

Right iliac fossa (RIF) mass is a common condition seen by surgeons and involves diagnostic challenges in making correct preoperative diagnosis. Though appendicular mass is one of the most common diagnosis, ileo-cecal tuberculosis (TB), cecal carcinoma, nonHodgkin's lymphoma, iliopsoas abscess, Chron's disease, urologic and gynecologic masses are other rare causes $(1,2)$. Despite advances in diagnostic tools, RIF remains a diagnostic and therapeutic challenge, hence many authors describe it as a temple of surprises. This report presents a challenging case of a RIF mass which turned out to be an appendicular mass caused by a fish bone.

\section{Case presentation}

A 35-year-old man, known case of hypertension and bronchial asthma, presented with one-month history of non-radiating RIF pain, associated with two days of vomiting. He did not have fever, and denied any foreign body ingestion, abdominal trauma, or constitutional symptoms. He was generally well and was not septic or anemic. Per abdomen examination revealed tenderness 
and guarding at the RIF with a palpable mass measuring $4 \mathrm{~cm} \times 4 \mathrm{~cm}$. Biochemical markers showed leukocytosis $\left(13,280 / \mathrm{mm}^{3}\right)$, capsular-polysaccharide reactive protein (CRP) of $128 \mathrm{mg} / \mathrm{L}$, and erythrocyte sedimentation rate (ESR) of $95 \mathrm{~mm} / \mathrm{hour}$.

Abdominal ultrasonography (USG) was suggestive of appendicular abscess. Computed tomography (CT) scan abdomen/pelvis showed a RIF mass which had no clear fat plane with adjacent transversus abdominis muscle, cecal pole and terminal ileum. The appendix was not visualized, and there was no foreign body noted (Figure 1). He was treated non-surgically with antibiotic for appendicular mass and responded well to treatment. He was discharged with a colonoscopy appointment in a month.

The colonoscopy showed non-distensible cecum secondary to external compression with a polypoidal growth at the appendiceal opening. Histopathology examination (HPE) of the mass showed inflamed stromal tissue with mucin lake, but no malignant cells. In view of the suspicion of neoplasm, we arranged an early clinic appointment after the colonoscopy to discuss further intervention with patient; however, he defaulted on the follow up.

One month after the colonoscopy, he presented again with the same abdominal pain. This time, the RIF mass was clinically larger, measuring $7 \mathrm{~cm} \times 7 \mathrm{~cm}$. He was also investigated for tuberculosis. Mantoux test, acid fast bacilli sputum culture and smear, and TB polymerase chain reaction assay were sent, and confirmed negative for TB.

A CT abdomen/pelvis was repeated, which revealed a larger right iliac mass compared with the first CT scan. A suspicious foreign body within the appendicular mass was visible in the repeat CT scan (Figure 2), which was presumed to be not visible in the previous CT scan. The initial CT scan was reviewed again by the surgeons and radiologists in a multi-departmental conference and different planes (coronal and sagittal) were revised meticulously. To our surprise, the sagittal plane of the initial CT scan did reveal a suspicious thin, elongated foreign body within the appendicular mass (Figure 3), which was not clearly visualized in axial and coronal planes.
A diagnostic laparoscopy was done. Intraoperatively, the terminal ileum, cecum and omentum were densely adherent to the lateral abdominal wall. There was difficulty in identifying the appendix laparoscopically due to dense adhesion. Laparotomy was done and on mobilizing the caecum posteriorly, there was a small cavity with pus collection, containing a 4-cm long foreign body which appeared to be like a fish bone within macerated appendix (Figures 4-6). Right hemicolectomy and ileo-colic anastomosis were done. Postoperative recovery was uneventful, and the HPE confirmed the tissue as perforated appendicitis.

\section{Discussion}

Foreign body ingestion was reported centuries ago. The most common foreign bodies ingested are fish bones, and can cause perforation most commonly at terminal ileum, followed by second part of duodenum. However, in general, gastrointestinal tract perforation due to foreign bodies is rare, less than $1 \%$ (3).

Foreign body-induced appendicitis has been reported since 18 th century with overall incidence of $0.005 \%$ (4), but the foreign body causing an appendicular mass is extremely rare (4). In our case, we postulated that the weight of the fish bone was greater than the bowel fluid content, hence it arrested in the caecum and gravitated towards the appendiceal orifice, which was the most dependent part of the cecum. Once it entered the appendiceal lumen, it caused appendicitis, perforation, and subsequently progressed to appendicular mass.

Ultrasound is helpful in RIF mass as it can identify the origin of pathological organ in $97 \%$ of cases (6), but identifying a foreign body is challenging, especially if patient does not give history of foreign body ingestion. Plain radiograph has sensitivity of only $32 \%$ to detect fish bones due to obscuration by soft tissue and fluid, although fish bone are radio-opaque (3).

CT scan is the investigation of choice in many abdominal conditions, as it is objective and available in most centers nowadays. CT scan can delineate the organ of origin, associated complications and help in preoperative planning (3). However, our case demonstrated 

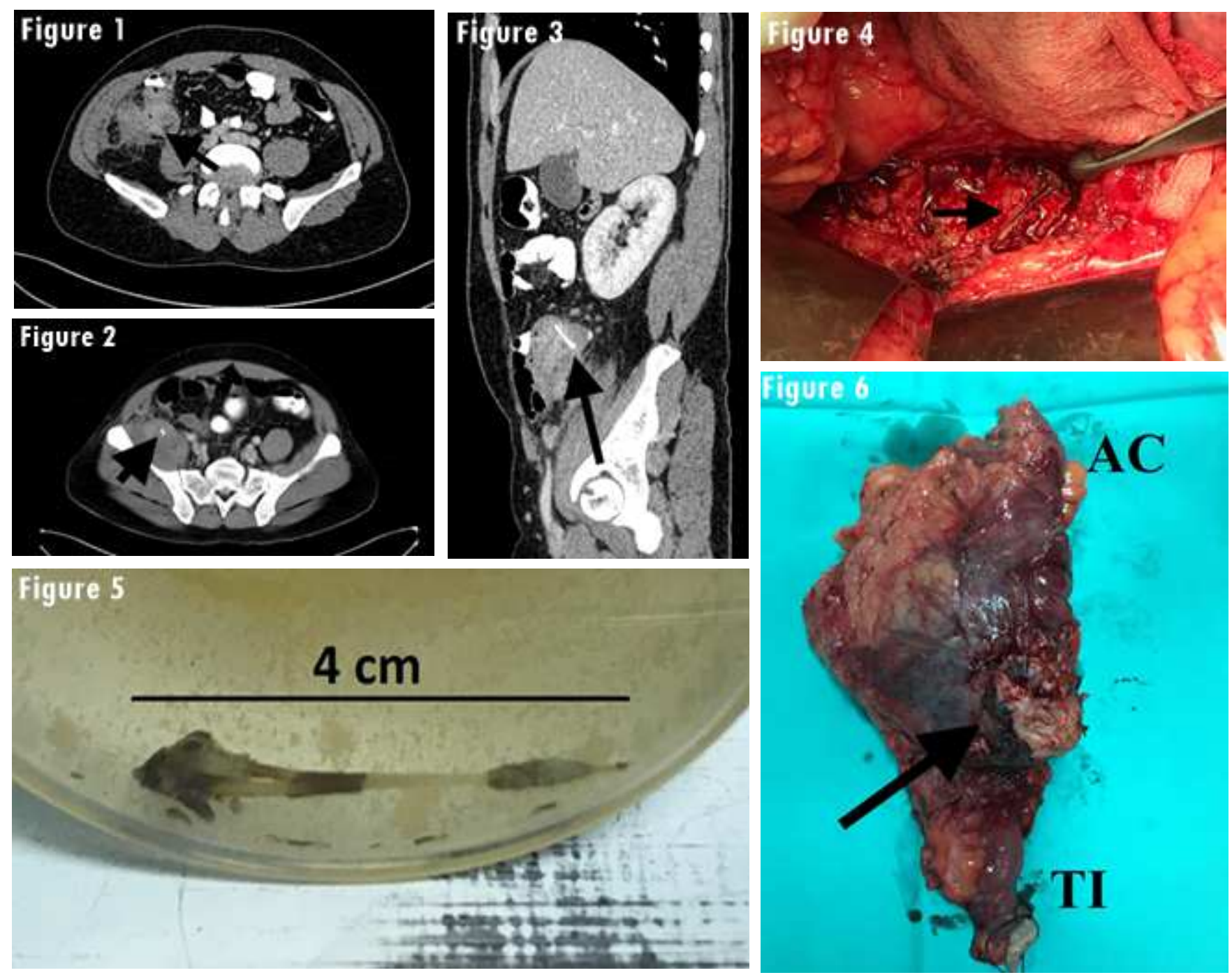

Figure 1: Axial cut of the first CT scan abdomen showing appendicular mass (arrow). No obvious foreign body can be visualized in this image; Figure 2: Axial cut of the repeated CT scan abdomen showing calcified foreign body (arrow) within the appendicular mass. Figure 3: Sagittal cut of the initial CT scan abdomen showing a suspicious thin elongated calcified foreign body (arrow) within the appendicular mass; Figure 4: Intraoperative image showing fish bone (arrow) in the cavity with macerated appendix; Figure 5: A fish bone measuring $4 \mathrm{~cm}$ in length retrieved from the macerated appendix; Figure 6: Posterior surface of the right hemicolectomy specimen showing the macerated appendix over the posterior cecal wall (arrow). AC - ascending colon, TI - terminal ileum.

that CT is not always a sensitive tool for fish bone detection. Fish bone may be obscured by intraluminal contrast media, or may mimic small blood vessels if intravenous contrast is given $(3,7)$. Meticulous review of different planes - axial, coronal and sagittal — use of thinner CT slices, helical CT scan and 3-dimension CT reconstruction can help detect fish bone $(3,7)$, as shown in this case. Common things are common, hence the initial diagnosis of appendicular mass in this case. When conservative management with antibiotic had failed, other important differentials were considered including ileo-cecal TB and cecal carcinoma $(1,2)$.

Colonoscopy is the gold standard when colon carcinoma is suspected. In this case, after ruling out pulmonary TB, colonoscopy was done which showed a tumor at the appendiceal opening. Although the HPE did not show any malignant cells, it did show inflamed stromal tissue with mucin lake, which raised the suspicion of 
neoplasm; however, patient defaulted on the follow-up to review the HPE and to discuss further intervention. In dealing with RIF mass, laparoscopy can be considered especially when CT scan and endoscopic findings are inconclusive. It can distinguish between appendicular abscess and mass, and is proved to be safe and feasible to treat appendicular mass (8). The management of RIF mass depends on the diagnosis. For appendicular mass, conservative treatment without interval appendicectomy is considered the best treatment by some authors (9). Other options include abscess drainage, nonsurgical treatment with delayed or interval appendicectomy, and right hemicolectomy $(9,10)$. So far, there is no guideline for management of appendicular mass caused by foreign body in the published English medical literature (3), hence its management is of case-by-case basis.

\section{Conclusion}

A surgeon must be aware of the variation of differential diagnosis for RIF mass, but common diagnosis should always be entertained. When facing diagnostic challenges in RIF mass, multi-modal diagnostic tools must be considered: ultrasound, CT scan, endoscopy, tissue biopsy and laparoscopy. Multi-disciplinary approach and a serial imaging with meticulous review of different planes may be helpful in delineating intraabdominal foreign body, which is evident in this case. It is important to look for appendix in spite of adhesions. Foreign body in the lumen of appendix can cause appendicitis and in rare case, can proceed to form an appendicular mass, which is challenging to treat.

\section{Ethical consideration}

All ethical principles were respected. Publication of this article is approved by the secretariat of National Institutes of Health (NIH), number KKM.NIHSEC.8004/4/1 Jld.66 (05).

\section{Conflict of interest}

The authors have no conflicts of interest to declare.

\section{Consent}

Written informed consent was obtained from the patient described for publication of the images and case detail.

\section{Financial disclosure}

The authors declared that this study has received no financial support.

\section{References}

1. Shashikala V, Alister JV, Sonia RPB. Right iliac fossa mass: a prospective study. Int J Biomed Adv Res. 2016; 7(8):388-392.

2. Rani KU, Shastri RK, Kapu H. Right iliac fossa mass: a case series. J Dent Med Sci (IOSR-JDMS). 2017; 16(3):11-14.

3. Beh JCY, Uppaluri AS, Koh BFJ, et al. Fishbone perforated appendicitis. J Radiol Case Rep. 2016; 10(7):14-22.

4. Klingler PJ, Seelig MH, DeVault KR, et al. Ingested foreign bodies within the appendix: a 100-year review of the literature. Dig Dis. 1998; 16:308-314.

5. Sar S, Kamal KM, Marsh R, et al. Recurrent appendicitis following successful conservative management of an appendicular mass in association with a foreign body: a case report. Cases J. 2009; 2:7776. 6. Millard FC, Collins MC, Peck RJ. Ultrasound is the investigation of the right iliac fossa mass. Br J Radiol. 1991; 64(757):17-19.

7. Beecher SM, O'Leary DP, et al. Diagnostic dilemmas due to fish bone ingestion, case report \& literature review. Int J Surg Case Rep. 2015; 13:112-115.

8. Vishwanath VS, Thinakaran $\mathrm{K}$, Rao TN, et al. Laparoscopic management of appendicular mass. J Minim Access Surg. 2011; $7(2): 136-140$

9. Tannoury J, Abboud B. Treatment options of inflammatory appendiceal masses in adults. World J Gastroenterol. 2013; 19(25):3942-3950.

10. Zerem E, Kunosic' S, Handanagic' A, et al. Minimally invasive treatment for appendiceal mass formed after acute perforated appendicitis. Surg Laparosc Endosc Percutan Tech. 2017; 22:3. 